
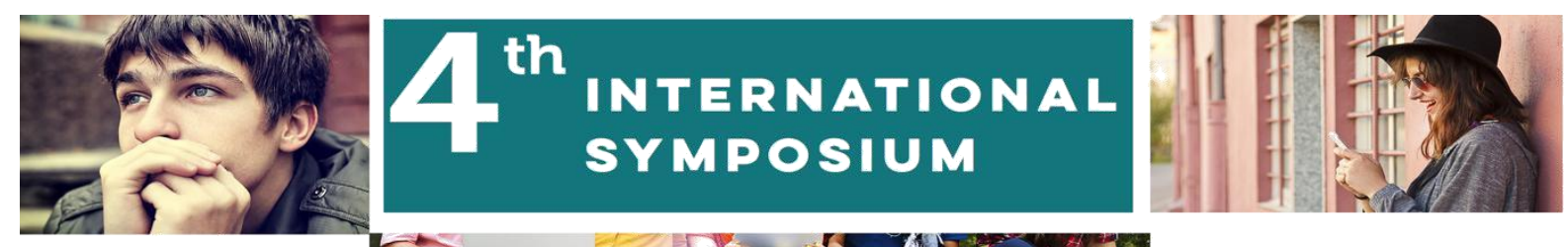

UNIFESP

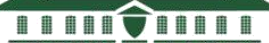

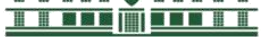

UNIVERSIDAPE FEDERAL DE SÁO PAỤLO

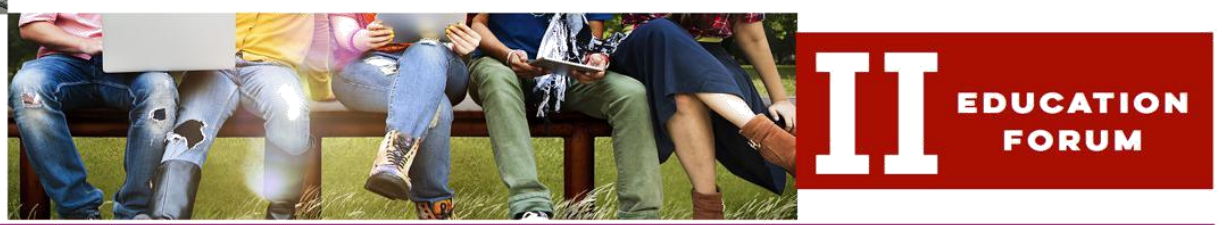

Adolescence(s): Vulnerabilities, Protagonisms and Challenges

INTERVENÇÕES COM PAIS EM UNIDADE DE TERAPIA INTENSIVA: UMA REVISÃO DA LITERATURA

\title{
INTERVENTIONS WITH PARENTS IN INTENSIVE THERAPY UNIT: A REVIEW OF THE LITERATURE
}

DOI: $\underline{\text { https://doi.org/10.22388/2525-5894.2018.0074 }}$

Mariana Batista Claudino - Aluna da Faculdade de Psicologia da Pontifícia Universidade Católica de Campinas, bolsista FAPIC/Reitoria.E-mail: marianabclaudino@gmail.com

Helena Bazanelli Prebianchi - Docente do Programa de Pós-Graduação em Psicologia e da Faculdade de Psicologia da Pontifícia Universidade Católica de Campinas. E-mail: helenabp@puc-campinas.edu.br

Projeto de Iniciação Científica pertencente ao Grupo de Pesquisa Institucional Pesquisas em Saúde e Desenvolvimento da Criança e Adolescente, da Pontifícia Universidade Católica de Campinas. 


\title{
RESUMO
}

O desenvolvimento da ciência médica tem possibilitado salvar e prolongar a vida de pacientes de todas as idades. Contudo, o ambiente frio e hostil das Unidades de Terapia Intensiva (UTI), pode ser traumático para pacientes infantis e suas famílias. Estudos sobre a família da criança hospitalizada podem fornecer subsídios para a reflexão e redirecionamento das práticas de cuidado em unidades pediátricas e neonatais. $\mathrm{O}$ objetivo deste trabalho foi investigar, através da revisão da literatura, as vivências e estados emocionais de pais/cuidadores de crianças internadas em UTIs neonatais e pediátricas e os programas de intervenção junto a eles. Os trabalhos publicados no período de 2010 a 2016, foram selecionados em indexadores de produção científica (BIREME, MedLine, PsycINFO, Scielo, LILACS). Dezoito artigos foram encontrados. A análise dos resultados indicou a presença, nos pais, de estresse, ansiedade, angústia, medo e depressão e de intervenções eficazes para redução desses sintomas. Discutiu-se a relevância dos estudos analisados, a escassez de trabalhos nacionais e a pouca frequência de estudos no contexto das UTIs. Concluiu-se sobre a necessidade de novos trabalhos que preencham as lacunas existentes e instrumentalizem a equipe.

Palavras-chaves: Unidade de Terapia Intensiva Pediátrica; saúde da criança; pais.

\begin{abstract}
The development of medical science has made it possible to save and extend the lives of patients of all ages. However, the cold and hostile environment of Intensive Care Units (ICUs) can be traumatic for children and their families. Studies on the family of hospitalized children can provide support for the reflection and redirection of care practices in pediatric and neonatal units. The objective of this study was to investigate, through the literature review, the experiences and emotional states of parents / caregivers of children hospitalized in neonatal and pediatric ICUs and the intervention programs with them. The works published in the period from 2010 to 2016 were selected in indexes of scientific production (BIREME, MedLine, PsycINFO, Scielo, LILACS). Eighteen articles were found. The analysis of the results indicated the presence, in the parents, of stress, anxiety, anguish, fear and depression and of effective interventions to reduce these symptoms. The relevance of the studies analized and the scarcity of national studies and the low frequency of studies in the context were discussed. It was conclude on the need for new works that fill the existing gaps and instrumentalize the team.
\end{abstract}

Key-words: Pedriatric Intensive Care Unit; child health, parents. 


\section{INTRODUÇÃO}

Comumente, a internação de uma criança na Unidade de Terapia Intensiva (UTI) interfere na dinâmica entre os envolvidos nesta situação, pois, o adoecimento afeta a rede de apoio do indivíduo e, no caso da criança, afeta, principalmente, seus cuidadores primários. (BARROS, 1999; GUIMARÃES, 1998; QUILES \& CARRILLO, 2000; SCOTT \& ARSLANIAN-ERGOREN, 2005).

Do ponto de vista psicológico, a experiência de adoecimento envolve inúmeras perdas transitórias e/ou permanentes e quando se faz necessária a internação hospitalar, o caráter potencial estressante do adoecimento é frequentemente aumentado (Doca \& Costa Junior, 2007). Quando o paciente internado é uma criança, as perdas e fatores estressantes afetam, mais diretamente, seus familiares. Estes, por sua vez, podem afetar o estado emocional da criança e seu repertorio de comportamentos diante da doença e do tratamento, especialmente quando o adulto é a mãe, o pai ou pessoa de referência pessoal da criança (COSTA JUNIOR, 2004; DOCA \& COSTA JUNIOR, 2007).

Levando em consideração que os pais exercem influência significativa no comportamento dos filhos, eles também requerem orientação e preparação para hospitalização das crianças. Quando a situação da criança se agrava e a família recebe a notícia da necessidade de internação na UTI, geralmente surge uma sensação de estranheza e impotência, carregada de stress, ansiedade e temor, pois a família além de fazer uma correlação imediata entre UTI e morte, também considera a separação temporária do filho muito dolorosa (BARROS, 2010).

As Unidades de Terapia Intensiva Neonatal (UTIN) e Pediátrica (UTIP) foram criadas com o propósito de salvar a vida de crianças em risco iminente de vida. Com o desenvolvimento da ciência médica, junto à realização de procedimentos cada vez mais complexos e por vezes invasivos, aliados à utilização de tecnologias cada vez mais potentes, têm conseguido salvar e prolongar a vida de pacientes de todas as idades. Porém, o ambiente frio e hostil dessas unidades traz traumas irreparáveis para a criança e para a família, principalmente quando é negada a esta o direito de permanecer junto a seu filho (MOLINA et al., 2009).

No cotidiano das instituições hospitalares e, principalmente nas UTINs e UTPs os profissionais envolvidos na assistência, vêm adotando um modelo centrado na criança e na família (COSTA, SANTOS, VILA BOAS, 2007). Constituem princípios básicos de cuidado centrado na família a permissão para uma presença irrestrita dos familiares na Unidade de Cuidados Intensivos Neonatais, sua participação nos cuidados da criança e uma comunicação aberta dos profissionais com os pais.

A presença de um familiar geralmente promove e mantém a inter-relação da criança com a família, neutraliza os efeitos decorrentes da separação, colabora na assistência integral à criança, melhora sua adaptação ao hospital, facilita a aceitação do tratamento, promove a positiva resposta terapêutica, ameniza os fatores estressantes da doença, dos procedimentos e da hospitalização (RIBEIRO, 2004). Além disso, a liberação 
da permanência da família na UTIN ou UTIP, durante a hospitalização do filho é uma estratégia que possibilita a redução do estresse emocional tanto da criança como da família, ao mesmo tempo em que contribui para diminuir o tempo de internação. (MOLINA et al., 2007).

Contudo, estudos como o de Darbyshire (2003), por exemplo, apontam grande variabilidade no conhecimento e entendimento dos pais sobre a hospitalização de seus filhos, com relatos indicando que parcela significativa dos pais não sabia, ou não tinham sido informados, sobre o que esperar do processo de internação. Em estudo realizado com equipe de profissionais de três Unidades de Terapia Intensiva, Roseiro e Paula (2015), os participantes apontaram a importância da promoção de ações direcionadas ao "cuidado do cuidador", que contemplem a criação de espaços onde eles possam expressar suas vivencias das situações cotidianas nas UTIs e receberem treinamento para manejarem situações estressantes.

Uma abordagem humanizada, que considere e respeite as características tão peculiares dessa clientela e situação, parece constituir-se no primeiro passo para que as famílias consigam lidar adequadamente com a internação de seus filhos na UTIN ou na UTIP, transformando esse desafio em uma oportunidade de aprendizagem e desenvolvimento pessoal. (MOLINA et al., 2007). Estudos sobre a família da criança hospitalizada podem fornecer subsídios para a reflexão e redirecionamento das práticas de cuidado em unidades pediátricas e neonatais e, também, valorizar as expectativas e opiniões desses pais em relação à atenção que recebem da equipe hospitalar.

Nesse sentido, o presente trabalho objetivou investigar, através da revisão da literatura, as vivências e estados emocionais de pais/cuidadores de crianças internadas em UTIs e UTIPs e os programas de intervenção junto a eles.

\section{MÉTODO}

Adotou-se o referencial metodológico da pesquisa bibliográfica, priorizando um caráter retrospectivo com abordagem quantiqualitativa. A seleção de trabalhos foi feita em indexadores de produção cientifica (BIREME, MedLine, PsycINFO, Scielo, LILACS). Para a busca, foram determinados os seguintes descritores em português: "pais", "cuidadores", "treino parental", "UTI neonatal", "UTI pediátrica”, "intervenção", "depressão", "estresse" e em inglês: "parentes", "caregivers", "parental training", "neonatal ICU", "pediatric ICU", "intervention", "depression", "stress".

Os critérios de inclusão foram: ser um trabalho publicado no período d 2010 a 2016 e que apresentasse relação com o tema e objetivos do estudo. Os critérios de exclusão foram: todos os artigos científicos publicados no período de 2010 a 2016, que não apresentassem relação com o tema ou fossem revisões bibliográficas, teses ou dissertações.

\section{Procedimentos de análise}

De acordo com a natureza e objetivos da pesquisa, os trabalhos selecionados foram codificados em cinco critérios para análise: objetivos, método, local da pesquisa, apresentação dos resultados e aspectos emocionais focalizados. 


\section{RESULTADOS E DISCUSSÃO}

Inicialmente, foram identificados 102 estudos, encontrados nos seguintes indexadores: BIREME ( $n=92)$; Scielo $(n=1)$, LILACS $(n=9)$. Dentre esses trabalhos identificados, foram removidos 21 artigos por estarem duplicados. Foram lidos 81 resumos e por critério de exclusão, foram eliminados 63 artigos. Após refinamento, foram selecionados 18 artigos, os quais foram lidos integralmente e incluídos na análise. Os procedimentos de etapas de avaliação e de obtenção dos trabalhos utilizados estão ilustrados na Figura 1.

Figura 1. Etapas de avaliação e obtenção dos trabalhos
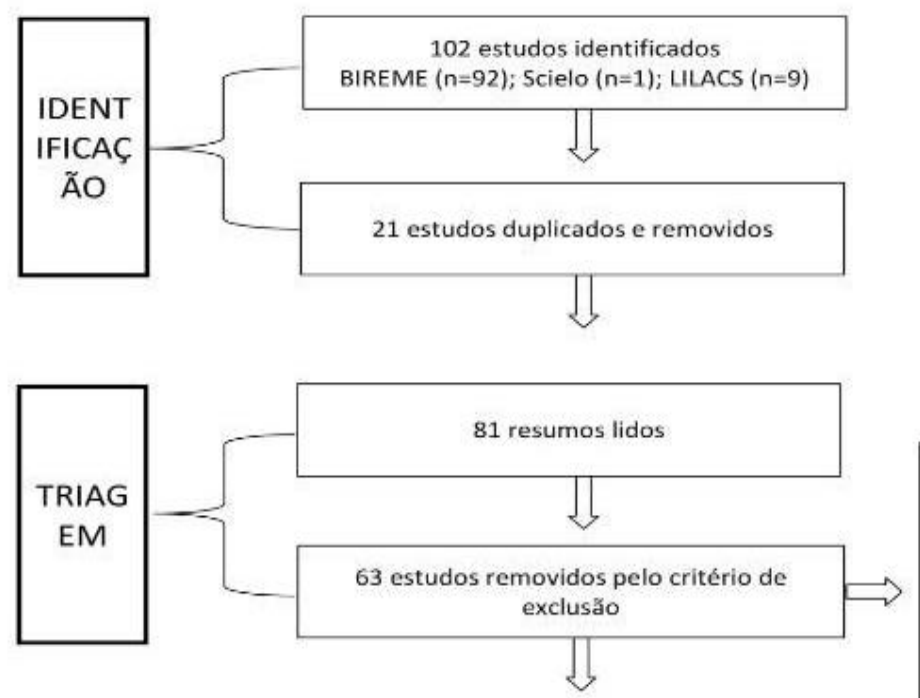

(i) Fora do periodo selecionado

(2010/2016);

(ii) Nå apresentaram relação

(iii) Trabalhos com texto completo em outro idioma além do português e inglês.

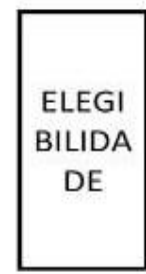

18 estudos selecionados para leitura integral

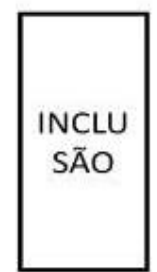

18 estudos incluidos para análise 
Tabela 1. Lista de artigos identificados na revisão sistemática com informações sobre o primeiro autor, título, ano e periódico.

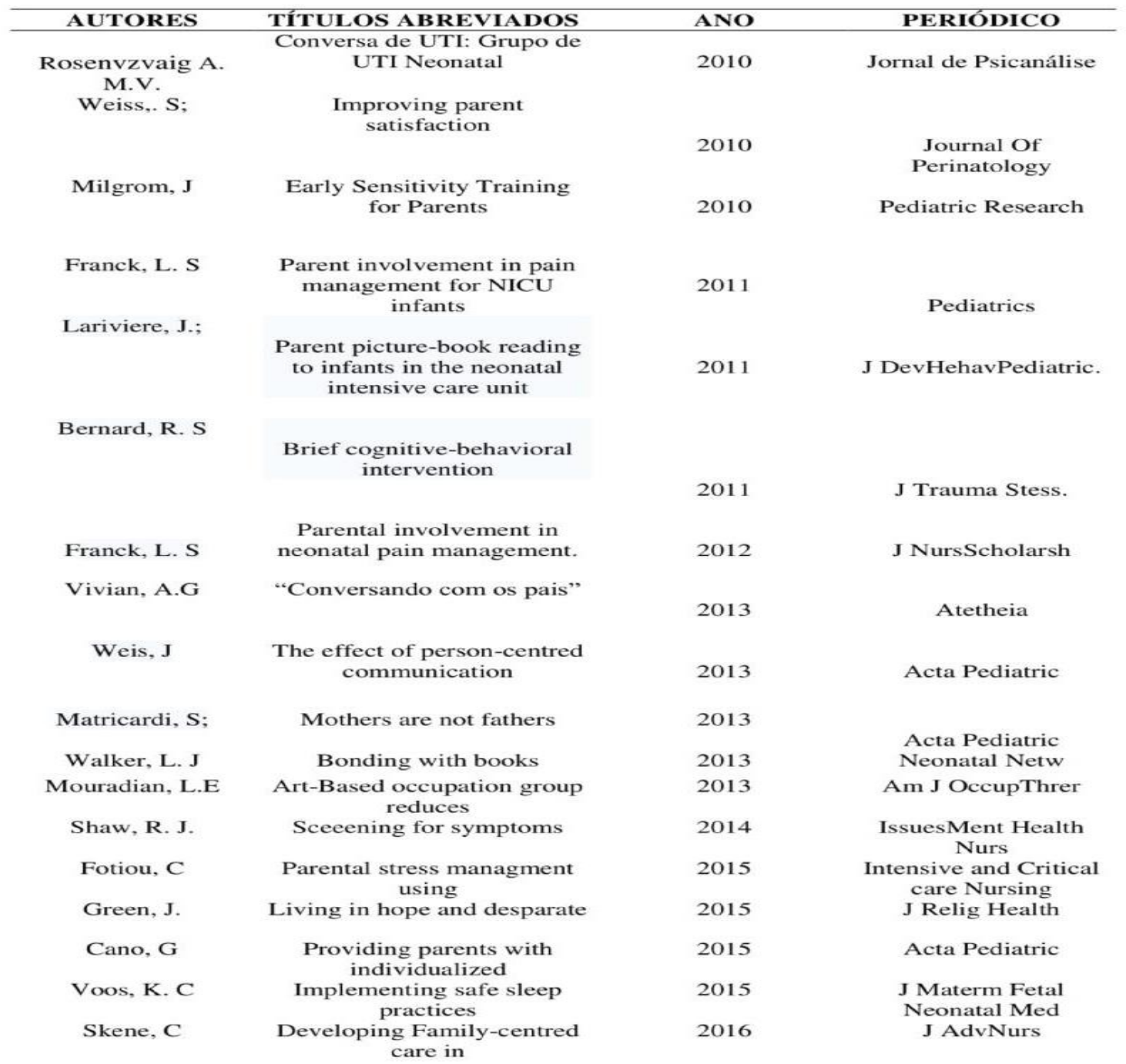

A Tabela 1, apresenta as principais características dos artigos selecionados. Com relação aos anos de publicação, foram selecionados 3 artigos de 2010 (Rosenzvaig, 2010. Weiss; Goldlust; Vaucher, 2010. Milgrom et al.,2010), 3 artigos de 2011 (Franck et al., 2011. Lariviere; Rennick, 2011. Bernard et al., 2011), 1 artigo do ano de 2012 (Franck; Outlon; Bruce, 2012), 5 de 2013 (Vivian et al., 2013. Weis et al., 2013. Matricardi et al., 2013. Walker, 2013. Mourandian; DeGrace; Thompson, 2013), 1 de 2014 (Shaw et al.,2014), 4 (Fotiou; Vlastarakos; Bakoula; Papagaroufalis; Bakoyannis; Darviri; Chrousos, 2015. Green, 2015. Cano; Sánchez-luna, 2015. Voos et al.,2015) e um de 2016 (Skene et al.,2016). É valido ressaltar que 16 artigos lidos (Bernard, et al.,2011. Cano; Sánchez-luna, 2015. Franck et al., 2011. Franck; Outlon; Bruce, 2012. Fotiou et al., 2015. Green, 2015. Lariviere; Rennick, 2011. Matricardiet al.,2013. Milgrom, et al., 2010. Mourandian; DeGrace; Thompson, 2013. Shaw, et al., 2014. Skene et al., 2016. Voos et al., 2015. Walker, 2013. Weiset al., 2013. Weiss; Goldlust; aucher, 2010) se referem a trabalhos na língua estrangeira e apenas 2 trabalhos (Rosenzvaig, 2010. Vivian et al., 2013) são da língua portuguesa.

Os resultados foram categorizados em cinco critérios para análise: objetivos, método do estudo, local de pesquisa, apresentação dos resultados e aspectos emocionais focalizados. Foi possível observar que todos 
os artigos selecionados tiveram como objetivo confirmar a eficácia de intervenções propostas, além de compreender sua contribuição aos participantes.

Treze trabalhos analisados tiveram como objetivo, intervenções que se basearam na redução dos sintomas de aspectos emocionais. No estudo de Bernard et al., (2011), foi possível identificar os aspectos emocionais: depressão e sintomas traumáticos. Já no estudo de Cano; Sánchez-luna (2015) os aspectos emocionais analisados foram depressão e ansiedade. $O$ estresse, foi aspecto emocional focalizado nos estudos de Franck et al., (2011), Matricardi et al., (2013), Milgrom et al,(2010), Weis et al., (2013) e Weiss; Goldlust; Vaucher, (2010).

Fotiouet al., (2015), Mourandian; DeGrace; Thompson, (2013) e Vivian et al., (2013) focalizaram as intervenções em aspectos emocionais como ansiedade e estresse. Green (2015) focou o estudo em aspectos de medos e angústias. Rosenzvaig (2010) focou o estudo no sofrimento vivenciado pelos pais, os medos e angustias por estarem no contexto de UTI Neonatal. O trabalho de Shaw et al., (2014) focou em aspectos emocionais relacionados à depressão, ansiedade e estresse pós-traumático.

Foi possível constatar que três estudos (FRANCK; OUTLON; BRUCE, 2012. FRANCK et al., 2011. SKENE et al., 2016) tiveram também como objetivo em comum, construir um maior conhecimento dos pais quanto ao envolvimento no manejo da dor infantil no ambiente de UTIN, além de promover o envolvimento dos pais no cuidado de bebês. Já os trabalhos de Lariviere; Rennick (2011) e Walker (2013), tiveram como objetivo examinar os efeitos de uma intervenção de leitura na UTIN como instrumento que pode conectar na interação pai-bebê. O estudo realizado por Voos et al., (2015), teve como objetivo desenvolver um modelo educacional de sono seguro para UTIN, devido a Síndrome da morte súbita do lactante ser a principal causa de morte no período pósnatal.

Quanto ao método utilizado, os resultados indicaram que dentre os 18 artigos analisados, 14 eram quanti-qualitativos: Bernard, et al., (2011). Cano; Sánchez-luna, (2015). Francket al. (2011). Fotiou et al., (2015). Lariviere; Rennick, (2011). Matricardi et al., (2013). Milgromet al., (2010). Mourandian; DeGrace; Thompson, (2013). Shaw et al., (2014). Skene et al., (2016). Vivian et al., (2013). Voos et al., (2015). Weis et al., (2013). Weiss; Goldlust; Vaucher, (2010). e que os demais 4 estudos: Franck; Outlon; Bruce, (2012). Green, (2015). Rosenzvaig, (2010). Walker, (2013), tratavam de pesquisas qualitativas.

Na pesquisa realizada por Fotiou et al. (2015) utilizou-se também técnica de relaxamento para avaliar a redução do nível de estresse/ansiedade. No estudo de Lariviere; Rennick (2011), foram usados também como metodologia, os efeitos da leitura de livro pelo pai para os filhos hospitalizados. E por fim, na pesquisa de Mourandian; DeGrace; Thompson (2013), foi utilizado o scrapbooking como instrumento para operacionalizar a ansiedade.

Analisando os estudos, 8 deles: Franck et al., (2011). Fotiou et al., (2015). Green, (2015). Lariviere; Rennick, (2011). Matricardi et al., (2013). Mourandian; DeGrace; Thompson, (2013). Shaw et al., (2014). Weis et al., (2013), também aplicaram instrumentos que avaliasse aspectos emocionais dos participantes, como estresse, ansiedade. Além disso, buscaram compreender como os resultados destas avaliações poderiam beneficiar os pais/cuidadores de crianças na UTIN e UTIP.

Com relação aos instrumentos utilizados, quatro estudos: Franck et al., (2011). Fotiou et al., (2015). Matricardi et al., (2013). Weis et al., (2013), basearam-se na utilização de instrumentos que avaliassem o nível de estresse dos pais com prematuros hospitalizados. Os estudos de Franck et al, (2011), Matricardi et al., (2013) e Weis et al., (2013) aplicaram a Escala "Parent Stressor Scale", sendo que o primeiro fez uso do instrumento somente após a intervenção. Na pesquisa realizada por Fotiou et al., (2015), foi utilizado a Escala "Perceived Stress Scale". 
Três estudos utilizaram inventários. Os estudos de Fotiou et al., (2015) e Mourandian; DeGrace; Thompson (2013), fizeram o uso do "State-TraitAnxietyInventory1and 2". O trabalho de Shaw et al., (2014) utilizou o inventario "Beck Depression Inventory (2a ed)". Foi possível observar que apenas um dos estudos (GREEN, 2015), utilizou entrevista semiestruturada. Além disso, três estudos, Green (2015). Lariviere; Rennick (2011). Shawet al., (2014), fizeram uso de questionário para os pais responderem. O trabalho de Shaw et al., (2014) utilizou o "Stanford Acute Stress Reaction Questionnaire".

Foi possível analisar que os 18 artigos abordaram intervenções psicológicas, sendo que 17 focalizaram em intervenções grupais, Bernard et al., (2011). Franck; Outlon; Bruce, (2012). Franck et al., (2011). Fotiou et al., (2015). Green, (2015). Lariviere; Rennick (2011). Matricardi et al., (2013). Milgrom et al., (2010). Mourandian; DeGrace; Thompson, (2013). Rosenzvaig (2010). Shaw et al., (2014). Skene et al. (2016). Vivian et al., (2013). Voos et al., (2015). Walker, (2013). Weis et al., (2013). Weiss; Goldlust; Vaucher, (2010) e 1 estudo focou em intervenção individual (CANO; SÁNCHEZ-LUNA, 2015).

$\mathrm{Na}$ intervenção realizada por Bernard et al., (2011), Cano; Sánchez-luna (2015), Franck et al., (2011), Fotiou et al., (2015), Lariviere; Rennick (2011), Matricardi et al., (2013), Milgrom et al., (2010) e Weis et al., (2013), os participantes foram selecionados e designados aleatoriamente para o grupo de controle e intervenção. Já nos estudos de Franck; Outlon; Bruce (2012), Green (2015), Shaw et al., (2014) e Walker (2013), todos participantes fizeram parte da intervenção.

Nas pesquisas realizadas por Mourandian; DeGrace; Thompson (2013) e Voos et al., (2015), a intervenção dividida em pré-atividade e pós-atividade, foi aplicada a todos os participantes. O estudo de Skene et al., (2016) foi dividido em três etapas (exploração, intervenção e avaliação) e todos os participantes fizeram parte da pesquisa. O estudo de Weiss; Goldlust; Vaucher (2010) foi dividido de forma semelhante, em três etapas: pré-intervenção, intervenção e pós-intervenção. Já nos estudos de Rosenzvaig (2010) e Vivian et al., (2013) a intervenção foi realizada com grupo de pais.

Com relação ao local da pesquisa, 17 trabalhos foram realizados em UTIN: Bernard et al., (2011). Cano; Sánchez-luna (2015). Franck; Outlon; Bruce (2012). Franck et al., (2011). Fotiouet al., (2015). Green (2015). Lariviere; Rennick (2011). Matricardi et al., (2013). Milgromet al., (2010). Mourandian; De Grace; Thompson (2013). Rosenzvaig, 2010.Shawet al., (2014). Skeneet al. (2016). Vooset al., (2015). Walker (2013). Weiset al.

(2013). Weiss; Goldlust; Vauche (2010.) e apenas 1 estudo ocorreu em UTIP (VIVIAN, et al., 2013).

No que se refere aos resultados dos estudos selecionados, pôde-se perceber que, os trabalhos de Bernard et al., (2011), Cano; Sánchez-luna, (2015), Fotiou et al., (2015), Matricardi et al., (2013) e Mourandian; DeGrace; Thompson (2013) apresentaram resultados positivos, pois concluíram que os sintomas de ansiedade, depressão e estresse foram reduzidos após a intervenção proposta. A pesquisa realizada por Franck; Outlon; Bruce (2012) teve como análise de seus resultados que o papel dos pais no manejo da dor infantil é um tema relativamente novo de pesquisa. Além disso, os autores mencionaram que este aspecto representa uma nova oportunidade para novos desenvolvimentos científicos com importantes implicações clinicas para o cuidado com os bebês críticos e suas famílias.

O estudo de Franck et al., (2011) focou no manejo da dor infantil e segundo os autores, os pais participantes valorizaram muito a atenção para a dor infantil e desejavam informações e envolvimento. Contudo, foi observado na pesquisa que os pais do grupo de intervenção estavam mais preparados que os pais do grupo de controle para assumir um papel ativo no tratamento da dor infantil, além de ter uma visão mais positiva sobre a realização de seu papel no período pós-alta.

A pesquisa de Green (2015), apresentou como resultados, o fato de que em algumas famílias, a esperança da intervenção divina parecia prevalecer e as enfermeiras eram testemunhas do desespero e do desapontamento das famílias quando um milagre não se aproximava. As intervenções realizadas com grupos 
de pais, nos estudos de Rosenzvaig (2010) e Vivian et al., (2013), apresentaram como resultados serem potencializadoras e agregadoras para os participantes das pesquisas. As autoras salientaram a forma que os pais se apropriavam da experiência, tirando dúvidas com os profissionais, além de compartilharem seus sentimentos, emoções, medos e angústias.

No estudo de Shaw et al., (2014), não foi positivo para o autor, diferenciar entre as mães participantes, que obtiveram resultados positivos e negativos diante das medidas utilizadas. A pesquisa realizada por Weis et al., (2013) também não foi capaz de demonstrar resultados significativos, sendo sugerido pelos autores, a reaplicação do projeto e também o acréscimo de instrumentos sensíveis à interação humana para melhor investigação do tema.

Analisando o estudo realizado por Skene et al., (2016), foi constatado que os resultados da pesquisa contribuíram com novos conhecimentos sobre abordagens para envolver ativamente os pais no cuidado de bebes em UTIN. Além disso, o artigo enfatiza a compreensão do impacto do cuidado centrado na família para ações políticas e práticas futuras.

Na intervenção realizada por Milgrom et al., (2010) não houve efeitos significativos em qualquer volume cerebral dos bebês prematuros. Além disso, concluiu-se que os resultados forneceram uma plataforma para consideração de maiores estudos randomizados de intervenções deste tipo em bebês prematuros. Os autores enfatizaram, ainda, a utilidade da ressonância magnética como um biomarcador precoce para lesão e desenvolvimento cerebral.

No trabalho de Voos et al., (2015) a intervenção aumentou a segurança no sono, além de aumentar a segurança do paciente. Os resultados das pesquisas de Lariviere; Rennick (2011) e Walker (2013) também foram positivos, sendo evidenciada que a leitura dos pais para bebês na UTIN é uma intervenção que pode conectar os pais e o bebê e oferece uma maneira de os pais participarem do cuidado.

Na pesquisa realizada por Weiss; Goldlust; Vaucher (2010) os participantes ficaram mais satisfeitos com a comunicação com o médico provedor na pós-intervenção do que na pré-intervenção. Concluiu-se que melhorando a qualidade e quantidade de comunicação dos pais com o médico, aumenta-se, consequentemente a satisfação dos pais com relação à comunicação deles com o médico de seus bebês.

De modo geral, 16 artigos analisados, Bernard et al., (2011). Cano; Sánchez-luna (2015). Franck; Outlon; Bruce (2012). Francket al., (2011). Fotiou et al., (2015). Green, 2015.Lariviere; Rennick, 2011. Matricardi et al., (2013). Milgrom et al., (2010). Mourandian; DeGrace; Thompson, (2013). Rosenzvaig, (2010). Skeneet al., (2016). Vivian et al., (2013). Voos et al., (2015). Walker (2013). Weiss; Goldlust; Vaucher (2010) apresentaram resultados positivos em suas pesquisas, tendo os objetivos alcançados. Somente 2 estudos Shaw, et al., (2014) e Weis et al., (2013) não apresentaram resultados satisfatórios, pois a metodologia da intervenção proposta foi considerada insuficiente.

No contexto de UTI Neonatal/Pediátrica, o objetivo primário é salvar vidas, focando o cuidado nos pacientes, sendo indispensável que a equipe de saúde, perceba as necessidades e prioridades dos hospitalizados e também de suas famílias. $\mathrm{O}$ avanço tecnológico da medicina, possibilitou o desenvolvimento de novos recursos que propiciaram a sobrevida das crianças e o prolongamento da vida de hospitalizado sem UTI Neonatal/Pediátrica (Saito, 2007).

A hospitalização precoce emocionalmente não só os bebês internados, mas também pais e cuidadores. Famílias que vivenciam este contexto podem ter como experiência períodos de desequilíbrio, instabilidade e fragilidade. Por isso é essencial que os pais dos hospitalizados enfrentem de forma positiva este momento, para que no final, estejam seguros e confiantes. Um aspecto facilitador no enfrentamento para famílias são as 
ações de apoio realizadas pela equipe, para que ajudem os envolvidos no enfrentamento, além de minimizar os desfechos psicológicos negativos que esta situação pode proporcionar (Cunha et al., 2017).

No Brasil, desde a criação do Estatuto da Criança e do Adolescente, em 1990, o qual determina a qualquer criança internada, o direito a um acompanhante em tempo integral (CORREA et al., 2015) a família passou a ser admitida no hospital. Especificamente, em ralação às UTINs, desde 2012, o Modelo do Cuidado Centrado no Paciente e Família (CCPF) tem sido adotado.

O CCPF aborda o planejamento, prestação e avaliação de saúde de modo a integrar a equipe de saúde, os pacientes e as famílias. Nesse Modelo a família é considerada central e constante na vida das pessoas como fonte primária de força e suporte e, portanto, deve-se assegurar que ela participe do planejamento das ações a serem tomadas com o paciente (BALBINO, BALIERO \& MANDETTA, 2016). Tendo isso em consideração, os resultados do presente estudo, revelando que apenas 2 dos trabalhos selecionados, são nacionais, sugerem que a produção científica da área, não reflete esses preceitos.

Talvez, isso se deva à formação deficitária, do psicólogo para a pesquisa e à falta de uniformização de conduta dos psicólogos atuantes em instituições hospitalares. O desconhecimento da necessidade da realização de pesquisas ou o despreparo para elas dificulta a divulgação da sua práxis e impede a verificação da efetividade das suas atuações e as repercussões dos serviços prestados.

Os pais exercem influência significativa no comportamento dos filhos, por isso eles também requerem orientação e preparação para a hospitalização das crianças. Segundo Barros (2010), um adulto calmo e seguro ajudam a criança a organizar-se e confiar; pais tensos e ansiosos e profissionais pouco preparados e igualmente ansiosos contribuem para aumentar a ansiedade da criança.

Os resultados encontrados evidenciam que essas preocupações têm norteado os trabalhos na área, pois a maioria dos estudos incluídos nessa pesquisa teve como objetivo a redução de sintomas psicológicos que afetam o bem-estar individual e as relações criança- cuidador, criança-equipe e família-equipe. As intervenções estudadas buscaram, de forma geral, diminuir o estresse, a ansiedade, a angústia e o medo dos pais e instrumentá-los para o manejo da dor infantil. (PADOVANI, et al.,2004).

O sucesso das intervenções confirma muitas asserções que têm sido feitas sobre o assunto. Saito (2007), afirma que uma ação conjunta entre a equipe multidisciplinar e os pais, para que juntos, possam ressignificar as vivencias nesta situação, transforma este contexto de hospitalização precoce em uma experiência positiva. Ainda no contexto da UTIN, Cunha et al., (2017), indica que através de condutas apropriadas e acolhedoras, o quadro aversivo pode ser desmistificado e transformado em uma experiência positiva para todos os envolvidos. Finalmente, Padovani et al., (2004), aponta para a importância de a equipe multidisciplinar oferecer intervenções suportivas para os familiares durante todo o percurso do tratamento dos bebês até a alta hospitalar, visando atender as necessidades psicológicas demandadas de forma preventiva para os participantes.

Além disso, as intervenções contribuíram para a satisfação dos pais com relação ao conhecimento obtido sobre o contexto da hospitalização de seus filhos, além de sentirem-se mais acolhidos pela equipe e preparados para o cuidado necessário com os bebês internados. Segundo Conner \& Nelson, (1999), a satisfação com os cuidados neonatais é um conceito complexo e englobam aspectos multidimensionais, como segurança, informações consistentes, comunicabilidade, educação, manejo da dor dos recém-nascidos, participação dos familiares nos cuidados, além do contato com o prematuro e apoio emocional. Compreender o grau de satisfação dos pais é importante, pois pode contribuir com informações úteis relacionadas às necessidades educacionais bem como com problemas ou deficiências nos cuidados oferecidos, além de retratar o sucesso ou falência do serviço de saúde (MEGIVERN; HALM; JONES, 1992). 


\section{CONSIDERAÇÕES FINAIS}

O presente estudo objetivou investigar por meio de uma revisão de literatura, as vivências e estados emocionais de pais e cuidadores de crianças internadas em UTI neonatal/pediátrica e os programas de intervenção junto a eles.

Os principais aspectos emocionais presentes nos artigos foram: depressão, ansiedade, estresse, medos, angustias, sintomas traumáticos.

Os resultados indicaram que as intervenções propostas nos estudos analisados foram, em geral, positivas, reduzindo os aspectos emocionais vivenciados pelos familiares, além de contribuir positivamente nas vivências dos pais participantes. Além disso, após as intervenções os pais se sentiram mais seguros e confiantes com relação ao contexto da hospitalização e prematuridade.

Contudo, os resultados também indicaram escassez de trabalhos nacionais publicados sobre o tema, características metodológicas limitadas dos artigos existentes e pouca informação sobre pais e crianças em UTIP. Preencher essas lacunas é essencial à compreensão das relações dos pais e cuidadores com as crianças hospitalizadas e com a equipe multidisciplinar, bem como para o desenvolvimento de intervenções que atendem e satisfaçam por completo as necessidades dos envolvidos neste contexto.

Assim, novos estudos são necessários a fim de se instrumentar cientificamente a prática das equipes de saúde e diminuir o sofrimento das crianças e seus familiares, minimizando desdobramentos futuros, negativos, em suas vidas.

\section{REFERÊNCIAS BIBLIOGRÁFICAS}

BALBINO, F. S., BALIERO, M. M. F. G., \& MANDETTA, M. A. Avaliação da percepção do cuidado centrado na família e do estresse parental em unidade neonatal. Rev. Latino-Am. Enfermagem, 24, 1-9, 2016. BARROS, L. Psicologia pediátrica: Perspectiva desenvolvimentista. Lisboa: Climepsi.1999.

BARROS, L. A dor pediátrica associada a procedimentos médicos: contributos da psicologia pediátrica. Temas em Psicologia, 18 (2), 295-306, 2010.

BERNARD, R. S. et al. Brief cognitive-behavioral intervention for maternal depression and trauma in the neonatal intensive care unit: a pilot study. J Trauma Stress, 24 (2), 230-234, 2011.

CANO, G. E.; SÁNCHEZ-LUNA, M. Providing parents with individualised support in a neonatal intensive care unit reduced stress, anxiety and depression. ActaPaediatr, 104 (7), 300-305, 2015.

CONNER, J. M., NELSON, E C. Neonatal Intensive Care: satisfaction measured from a parent's perspective. Pediatrics.p,336-349, 1999.

CORRÊA, A. R. et al. As práticas do Cuidado Centrado na Família na perspectiva do enfermeiro da Unidade Neonatal. Esc Anna Nery, 19(4), 629-634, 2015.

COSTA JUNIOR, A. L. A intervenção psicológica no contexto de procedimentos médicos invasivos em oncologia pediátrica: discussão metodológica: In M. Z. S. Brandão (Org.), Sobre comportamento e cognição Santo André, SP: Esetec, p. 139-151, 2004. 
COSTA, S. A. F., SANTOS, M. J. A., VILLAS BOAS, A. S. C. Compreendendo a vivencia da família com a internação do filho recém-nascido prematuro na UTI neonatal. Nursing, São Paulo, 115 (10), 560-4, 2007.

CUNHA, A. C. B. et al. Discutindo sobre estresse e enfrentamento da prematuridade por cuidadores. TES TemasemEducação e Saúde. 13 (1), 41-58, 2017.

DARBYSHIRE, P. Mother's experiences of their child's recovery in hospital and at home: A qualitative investigation. Journal of Child Health Care. 7, 291-312, 2003.

DOCA, F. N. P. \& COSTA JUNIRO, A. L. Preparação psicológica para admissão hospitalar de crianças: uma breve revisão. Paidéia, 17 (37), 167-179, 2007.

FRANCK, L. S. et al. Parent involvement in pain management for NICU infants: a randomized controlled trial. Pediatrics, 128 (3), 510-518, 2011.

FRANCK, L. S.; OULTON, K.; BRUCE, E. Parental involvement in neonatal pain management: an empirical and conceptual update. J Nurs Scholarsh, 44 (1), 45-54, 2012.

FATIOU, C. et al. Parental stress management using relaxation techniques in a neonatal intensive care unit: $\mathbf{A}$ randomised controlled trial. Intensive and Critical care Nursing, 20-27, 2015.

GREEN, J. Living in hope and desperate for a miracle: NICU nurses perceptions of parental anguish, J Relig Health, 54 (2), 731-744, 2015.

GUIMARÃES, S. S. A hospitalização na infância. Psicologia: Teoria e Pesquisa, 4, 102-112, 1988.

LARIVIERE, J.; Rennick, J. E. Parent picture-book reading to infants in the neonatal intensive care unit as an intervention supporting parent-infant interaction and later book reading. J Dev Behab Pediatr, 32 (2), 146-152, 2011.

MATRICARDI, S. et al. Mothers are not fathers: differences between parents in the reduction of stress levels after a parental intervention in a NICU. Acta Paediatr, 102 (1), 8-14, 2013.

MEGIVERN, K., HALM M. A., JONES. G. Measuring patient satisfaction as an outcome of nursing care. Journal of Nursing Care Quality, 6(4), 9-24, 1992.

MILGROM, J. et al. Early Sensitivity Training for Parents of Preterm Infants: Impact on the Developing Brain. Pediatric Research, 67(3), 330-335, 2010.

MOLINA, R. C. M. et al. A percepção da família sobre sua presença em uma Unidade de Terapia Intensiva Pediátrica e Neonatal. Rev. Esc Enferm. 43, (3). 630- 638, 2009.

MOLINA, R. C. M. et al. Presença da família nas Unidades de Terapia Intensiva Pediátrica e Neonatal: visão da equipe multidisciplinar. Esc Anna Nery Rev. Enferm. 11 (1), 437-44, 2007.

MOURADIAN, L. E.; DEGRACE, B. W.; THOMPSON, D. M. Art-based occupation group reduces parent anxiety in the neonatal intensive care unit: a mixed-methods study. Am J OccupTher, 67 (6), 692-700, 2013.

PADOVANI, F. H. P. et al. Avaliação de sintomas de ansiedade e depressão em mães de neonatos pré-termo durante e após hospitalização em UTI-neonatal. Rev. Bras. Psiquiatr, 26 (4), 251-254, 2004. 
QUILES, J. M. O. \& CARRILLO, F. X. M. Hospitalización infantil. Repercusiones psicológicas. Teoria y práctica. Madrid: Biblioteca Nueva, 2000.

RIBEIRO, N. R. R. A família enfrentando a doença grave da criança. In: Elsen, I., Marcon, S. S., Sillva, M. R. S. (organizadores). O viver em família e sua interface com a saúde e a doença. 2a ed. Maringá: Eduem, p. 183-97, 2004.

ROSEIRO, C. P \& Paula, K. M. P. Concepções de humanização de profissionais em Unidades de Terapia Intensiva Neonatal. Estudos de Psicologia. Campinas, 32 (1), 109-119, 2015.

ROSENZVAIG, A. M. V. Conversa de UTI: grupo de pais num serviço de UTI neonatal. J. psicanal. São Paulo, 43(79), p.163-169, 2010.

SAITO, A. Avaliação do grau de satisfação materna em UTI neonatal. Botucatu, 2007.Disponívelem: https://repositorio.unesp.br/handle/11449/96123.

SCOTT, L. D., \& ARSLANIAN-ERGOREN, C. The decision to care: A life-altering experience. Home Health Care Management \& Practice. 17, 130-135, 2005.

SHAW, R. J. et al. Screening for symptoms of postpartum traumatic stress in a sample of mothers with preterm infants. Issues Ment Health Nurs, 35 (3), p.198-207, 2014.

SKENE, C. et al. Developing family-centred care in a neonatal intensive care unit: an action research study protocol. J Adv Nurs, 72 (3), 658-668, 2016.

VIVIAN, A. G. et al. "Conversando com os pais": relato de uma experiência de intervenção em grupo em UTI pediátrica. Aletheia, 40, 174-184, 2013.

VOOS, K.C. et al. Implementing safe sleep practices in a neonatal intensive care unit. J Materm Fetal Neonatal Med, 28 (14), 1637-1640, 2015.

WALKER, J. L. Bonding with books: the parent-infant connection in the neonatal intensive care unit. Neonatal Netw, 32 (2), 104-109, 2013.

WEISS, J. et al. The effect of person-centred communication on parental stress in a NICU: a randomized clinical trial. Acta Paediatr, 102 (12), 1130-1136, 2013.

WEISS, S.; GOLDLUST, E.; VAUCHER, Y. E. Improving parent satisfaction: an intervention to increase neonatal parent-provider communication. Journal of Perinatology, 425-430, 2010. 